SLEDAI at active and remission phases were 4.25 vs 0.45 and 8.32 vs 1.25 , respectively. Fever, rash, and arthritis were the most common features and kidney was the most common involved organ at active phase. The mean serum complement factor $\mathrm{H}$ and I levels at active phase were significantly lower than that at remission phase. The mean serum CD46 level at active phase was higher significantly compared with that at remission phase. The serum $\mathrm{C} 5 \mathrm{a}$ and $\mathrm{C} 5 \mathrm{~b}-9$ at active and remission phases were no significant difference. Five patients had sequelae including 1 intracranial haemorrhage and 4 chronic kidney disease.

Conclusions Serum complement factor H, I and CD46, but not C5a and C5b-9 were associated with disease activity of SLE.

\section{THE ROLE OF PI3K, MTOR IN THE EXPRESSION OF INTERFERON -ALPHA INDUCED PROTEIN IFIT4 IN LUPUS}

${ }^{1} \mathrm{~J}$ Chen, ${ }^{2}$ X Huang*. 'Xiang Ya Second Hospital, Department of Rheumatology, chang sha, China; ${ }^{2}$ Xiang Ya Second Hospital, Rheumatology, chang sha, China

10.1136/lupus-2017-000215.280

Background and aims The role of Phosphoinositide 3-kinase (PI3K), mammalian target of rapamycin (mTOR) and dexamethasone in IFN- $\alpha$-induced-human interferon-induced protein with tetratricopeptide repeats 4 (IFIT4) expression was investigated.

Methods HT1080 cells were pre-treated with specific inhibitors of $\mathrm{PI} 3 \mathrm{~K} / \mathrm{mTOR}$, PKC or JNK transduction factors, then further incubated with IFN- $\alpha$ for different times. The mRNA and protein expression of IFIT4 or other indicated signal transduction factors were detected by qRT-PCR or westernblot.

Results LY294002, a dual mTOR and PI3K inhibitor, but not wortmannin, blocked IFIT4 promoter activation, mRNA and protein, as well as phosphorylation of STAT1, JNK, PKC $\delta$ induced by IFN- $\alpha$. Interestingly, rapamycin, mTOR inhibitor, had the same effects as LY294002, counteracting the IFN- $\alpha$ dependent upregulation of IFIT4 and phosphorylation of STAT1, JNK, PKCס. Rottlerin or Sp600125, specific inhibitor of PKC $\delta$, JNK, inhibited IFN-induced IFIT4 expression, but not the phosphorylation of AKT and mTOR. Interestingly, in vivo, bolus intravenous injection of methylprednisolone rapidly decreased the IFIT4 expression. In vitro, dexamethasone could prohibit IFN- $\alpha$-induced IFIT4 transcription and the phosphorylation of STAT1, JNK, PKC- $\delta$.

Conclusions IFN- $\alpha$ activate the PI3K and mTOR pathways, which converge to regulate $\mathrm{PKC} \delta$, JNK , STAT1-dependent transcription of IFIT4 in a mTOR dependent and AKT independent manner. The induction of IFIT4 transcription by IFN- $\alpha$ depends upon sequential activation of mTOR, PKC $\delta$, JNK and STAT1. Steriod might play the role in treatment for systemic lupus erythematosus (SLE) partially by the reason of decreasing IFN alpha induced protein IFIT4 expression via sequential inhibition of the phosphorylation of PI3K, mTOR, PKC $\delta$, JNK, STAT1.

\section{THE CLINICAL ANALYSIS OF THE CHARACTERISTICS OF LUPUS MESENTERIC VASCULITIS (LMV) COMPARED WITH LUPUS NEPHRITIS}

${ }^{1} \mathrm{~J}$ Chen, ${ }^{2} \mathrm{R}$ Wan, ${ }^{3} \mathrm{X}$ Rong, ${ }^{3} \mathrm{R}$ Wang, ${ }^{4} \mathrm{X}$ Huang ${ }^{*} .{ }^{1}$ Xiang Ya Second Hospital, Department of Rheumatology, chang sha, China; ${ }^{2}$ West China school of medicine- Sichuan University-, Department of Rheumatology, Si Chuan, China; ${ }^{3}$ West China Hospital- Sichuan University, Department of Rhematology, Si Chuan, China; ${ }^{4}$ Xiang Ya Second Hospital, Rheumatology, chang sha, China

\subsection{6/lupus-2017-000215.281}

Background and aims Evaluate the clinical characteristics of lupus mesenteric vasculitis (LMV) in a large cohort.

Methods The in-patients with Systemic Lupus erythematosus (SLE) admitted to the West China Hospital from 2009 to 2015 were analysed. Diagnosis of LMV was made according to the clinical symptoms, abdominal image and the reaction to steroids. The patients with Lupus Nephritis (LN) were set as the control. Qualitative differences were analysed using the chi-square test.

Results Among 3143 patients with SLE, 103 patients were diagnosed as LMV with the incidence of LMV being $32.8 \%$. Among those patients with LMV, 96.1\% was female and the average age was 35.8 years old, 37 (35.9\%) patients were diagnosed as SLE with LMV as the one of onset symptoms, $54(52.4 \%)$ patients were misdiagnosed at the first time. The incidence of abdominal pain was $87.4 \%$, while that of pelvic effusion $68.04 \%$, vomiting $70.9 \%$, bloating $68.6 \%$ and diarrhoea $67.0 \%$. Their average score of the SLE disease activity index (SLEDAI ) (including LMV as vasculitis ) was 21.8 \pm 7.7 . During the following-up for 2-96 months, partial remission of the incidence of abdominal pain within a week was $87.9 \%$, and $98.8 \%$ of the LMV patients got complete remission in a month. Eight cases experienced disease relapse (8.0\%). Rash $\left(X^{2}=6.7255, \quad \mathrm{p}=0.0095\right), \quad$ high creatinine $\left(X^{2}=6.746\right.$, $\mathrm{p}<0.0001)$, hypercholesterolemia $\left(\mathrm{X}^{2}=21.2986, \mathrm{p}<0.0001\right)$, leukopenia $\left(\mathrm{X}^{2}=7.4153, \mathrm{p}=0.0245\right)$ were presented more often in the patients with LMV than in LN.

Conclusions There were differences between LMV and LN in clinical symptoms and laboratory findings. A much lower mortality rate was found in LMV.

\section{EFFECT OF GLUTEN CONTAINING DIET ON PRISTANE INDUCED LUPUS PRONE MICE}

N Hussain*1 'University of the punjab, Microbiology and molecular genetics, Lahore, Pakistan

\subsection{6/lupus-2017-000215.282}

Background and aims SLE is a chronic autoimmune disease with characteristic organ involvement and autoantibodies production. The pathogenicity and aetiology of the disease yet to be elucidated. It is presently accepted that environmental factors trigger the disease in genetically sensitive individuals. Gluten, a protein fraction commonly found in wheat grains, associated with food related disorders and a number of autoimmune diseases. We hypothesised that gluten containing diet would further exacerbate an already undergoing arbitrary immune reaction in SLE patients. 